\title{
BMJ Open The impact of time-window bias on the assessment of the long-term effect of medication adherence: the case of secondary prevention after myocardial infarction
}

\author{
Mirko Di Martino, ${ }^{1}$ Ursula Kirchmayer, ${ }^{1}$ Nera Agabiti, ${ }^{1}$ Lisa Bauleo, ${ }^{1}$ Danilo Fusco, ${ }^{1}$ \\ Carlo Alberto Perucci, ${ }^{2}$ Marina Davoli ${ }^{1}$
}

To cite: Di Martino M, Kirchmayer U, Agabiti N, et al. The impact of timewindow bias on the assessment of the long-term effect of medication adherence: the case of secondary prevention after myocardial infarction. BMJ Open 2015:5: e007866. doi:10.1136/ bmjopen-2015-007866

- Prepublication history and additional material is available. To view please visit the journal (http://dx.doi.org/ 10.1136/bmjopen-2015007866).

Received 11 February 2015 Revised 29 April 2015 Accepted 7 May 2015

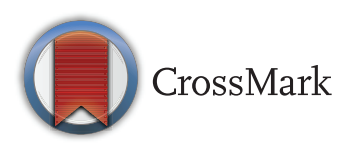

${ }^{1}$ Department of Epidemiology, Lazio Regional Health Service, Rome, Italy ${ }^{2}$ Senior Epidemiologist Consultant, Rome, Italy

Correspondence to Dr Mirko Di Martino; m.dimartino@deplazio.it

\section{ABSTRACT}

Objectives: Time-window bias was described in casecontrol studies and led to a biased estimate of drug effect. No studies have measured the impact of this bias on the assessment of the effect of medication adherence on health outcomes. Our goals were to estimate the association between adherence to drug therapies after myocardial infarction (MI) and the incidence of a new MI, and to quantify the error that would have been produced by a time-window bias.

Setting: This is a population-based study. Data were obtained from the Regional Health Information Systems of the Lazio Region in Central Italy (around 5 million inhabitants).

Participants: Patients discharged after MI in 2006-2007 were enrolled in the cohort and followed through 2009.

Outcome measure: The study outcome was reinfarction: either mortality, or hospital admission for MI, whichever occurred first.

Design: A nested case-control study was performed. Controls were selected using both time-dependent and time-independent sampling. Adherence to antiplatelets, $\beta$-blockers, ACE inhibitors/angiotensin receptor blockers (ACEI/ARBs) and statins was calculated using the proportion of days covered (PDC).

Results: A total of 6880 patients were enrolled in the cohort. Using time-dependent sampling, a protective effect was detected for all study drugs. Conversely, using time-independent sampling, the beneficial effect was attenuated, as in the case of antiplatelet agents and statins, or completely masked, as in the case of ACEI/ ARBs and $\beta$-blockers. For ACEl/ARBs, the time-dependent approach produced ORs of 0.83 ( $95 \% \mathrm{Cl} 0.57$ to 1.21$)$ and 0.72 ( 0.55 to 0.95 ), respectively, for ' $0.5<\mathrm{PDC} \leq 0.75$ ' and ' $P D C>0.75$ ' versus ' $0 \leq P D C \leq 0.5$ '. Using the timeindependent approach, the ORs were 0.96 (0.65 to 1.43) and 1.00 (0.76 to 1.33 ), respectively.

Conclusions: A time-independent definition of a timedependent exposure introduces a bias when the length of follow-up varies with the outcome. The persistence of time-related biases in peer-reviewed papers strongly suggests the need for increased awareness of this methodological pitfall.

\section{Strengths and limitations of this study}

No studies so far have measured the impact of time-window bias in evaluating the effect of medication adherence on health outcomes. We measured this impact by analysing the affect of evidence-based therapies for secondary prevention after myocardial infarction on the incidence of a new ischaemic heart attack.

- Population-based design, large numbers and robustness of analytical procedures are the main strengths. This study contributes to the debate on the complex methodology to estimate the effectiveness of medication in clinical practice.

- Our pharmaceutical database does not contain information on the prescribed daily doses, and adherence to drug treatment was estimated on the basis of the defined daily doses. Although this is a useful instrument for comparing the results from different studies, misclassification of drug utilisation may have occurred. However, both methods (time-independent and timedependent sampling) were performed and compared in the presence of the same conditions.

\section{BACKGROUND}

Randomised controlled trials are invaluable for assessing the efficacy of medications and obtaining regulatory approval. However, observational studies offer several advantages over clinical trials. A study based on a large and unselected population is more representative, and allows for greater generalisation. Of note, in clinical practice, some 'disturbing factors', such as the reduced adherence to chronic therapies, make therapeutic goals difficult to achieve. ${ }^{1}$ The availability of computerised health databases contributes decisively to observational studies of the impact of medications. However, the use of a nonexperimental setting to analyse the associations between drug exposures and health 
outcomes carries the risk of specific biases that may lead to erroneous results, especially when drug treatments and outcomes are both measured in the same time window.

Samy Suissa described the effect of the time-window bias in case-control studies. The bias was caused by the time-independent method used to select controls, who were defined as all the subjects who did not experience the study outcome during the observation period. Thus, the exposure was assessed during a shorter time interval for cases than for controls. ${ }^{2}$ This time-related bias can be easily avoided by using time-dependent sampling, according to the principle of incidence density sampling. ${ }^{3}$ This method assigns the same length of observation to cases and to their matched controls to ensure equal time windows to measure exposure. ${ }^{2}$

No studies have measured how the time-window bias affects assessments of the effect of medication adherence on health outcomes.

Our goals were to properly estimate the association between the adherence to evidence-based drug therapies for secondary prevention after an acute myocardial infarction (MI) ${ }^{4}$ and the incidence of a new MI, and to quantify the direction and magnitude of the error that would have been produced by time-window bias.

\section{MATERIALS AND METHODS Data sources}

Our department has access to regional health information systems that contain mortality, hospital admission and drug claims data. The details of the individual information systems are reported in the appendix.

\section{Setting and study cohort}

This population-based, observational, retrospective study was approved by the Internal Ethics Committee of the Department of Epidemiology, Regional Health Service, Lazio Region. The study was based on the population living in the Lazio region of Italy, which comprises approximately 5 million persons. Using data from the regional hospital information system, the study included a cohort consisting of all patients discharged from hospitals between 1 January 2006 and 30 November 2007 with a diagnosis of MI (index admission). An MI was defined as either a primary diagnosis of ICD-9-CM codes 410.xx or as a primary diagnosis of an MI-related condition along with a secondary diagnosis of 410.xx (see online supplementary appendix). Patients with a duration of the index admission $>30$ days (95th centile) were considered as outliers and excluded from this study.

Patients aged 35-100 years at discharge were considered for inclusion in the analysis. Only incident cases of MI were included. Patients with hospital admission during the previous 9 years for infarction, percutaneous coronary intervention (PCI), bypass, coronary disease or surgery of the heart and great vessels, were excluded. Patients who were not registered in the regional health assistance file were excluded because they could not be retrieved from the regional health information system (note that healthcare is offered to all resident citizens without restriction). Finally, patients who were hospitalised longer than half their individual follow-up interval or who received an outpatient regimen for less than 30 days were excluded because they were considered extremely complex or instable patients. The patient characteristics are described in detail elsewhere. ${ }^{6}$

\section{Follow-up and outcome}

Individual follow-up was considered to start on the first day after discharge from the index admission. The end of the observation period was defined as either the end of the study period (31 December 2009), the time of death not related to MI, or the date of an outcome, whichever occurred first. The study outcome was reinfarction, defined as either out-of-hospital mortality, ICD-9-CM 410-414, or hospital admission for MI, according to the inclusion criteria, whichever occurred first. ${ }^{7}$ The first 30 days after discharge were considered a buffer period to ensure that all patients had the chance to achieve clinical stability and obtain drug treatment, and to guarantee a minimum observation period of 1 month.

\section{Drug exposure}

Exposure information was collected from the regional registry of all drugs dispensed by public and private pharmacies (pharm); this registry is described in detail elsewhere. ${ }^{6}$ All drugs in this study were included in the patients' healthcare plans and are equally available to all residents through the universal healthcare coverage provided to residents of Italy.

The association between 'evidence-based' medication and outcome was analysed for the following drug treatments, identified through the anatomical therapeutic chemical (ATC) classification system: platelet aggregation inhibitors (ATC: B01AC04, B01AC05, B01AC06), $\beta$-blockers (ATC: C07), ACE inhibitors/angiotensin receptor blockers (ACEI/ARBs, ATC: C09), and statins (ATC: C10AA). Adherence was measured for each drug as the proportion of days covered (PDC) based on the defined daily doses (DDD). This approach was based on preliminary research. ${ }^{8}$ Adherence was categorised into the following three levels based on the PDC values: $0 \leq \mathrm{PDC} \leq 0.5 ; 0.5<\mathrm{PDC} \leq 0.75$; and $\mathrm{PDC}>0.75$. After preliminary analysis, we decided to use different thresholds for $\beta$-blockers (ie, 0.25 and 0.50 ). In fact, in clinical practice, $\beta$-blockers are prescribed at dosages lower than the DDD for secondary prevention after an MI. ${ }^{9}$ In order to reduce the 'immeasurable time bias' ${ }^{10}$ we assumed a daily drug intake of one DDD for inpatient regimens, since in Italy drugs are generally dispensed by the facility during inpatient treatment, and thus cannot be retrieved from the 'Pharm' registry. However, there is no clearly valid approach to data analysis that can definitively circumvent this bias. ${ }^{10}$ 
Study design and statistical analysis

The probability of drug adherence $(\mathrm{PDC}>0.75)$ and its 95\% CI was estimated as a function of the follow-up time, starting from the date of discharge of the index MI, using logistic regression models. A nested casecontrol study was performed to assess the relationship between adherence to treatment and outcome. All cases of reinfarction were included in the analyses. We selected the controls using two methods, the timedependent sampling and the 'biased' time-independent sampling, so that the effect of these two sampling methods could be compared. Up to four controls were individually matched to each case on sex and age (5-year groups). Median observation time (days) for cases and controls was reported. Conditional logistic regression was performed to measure the adherence effect for each of the study drugs. Potential confounders were selected in two steps. First, the following potential risk factors were selected based on a priori knowledge of the disease: the duration of the index admission, revascularisation procedures during the index admission (PCI or bypass), 17 comorbidities retrieved from the hospital records for both the index admission and the nine previous years (see online supplementary appendix), use of $\beta$-blocker, ACEI/ARB or statin medication during the 12 months prior to the index admission (defined as at least two prescriptions) and concomitant therapy with any of the other three evidence-based drug groups, classified as follows: no treatment, $0<\mathrm{PDC} \leq 0.75$ (0.50 for $\beta$-blockers) and PDC $>0.75 \quad(0.50$ for $\beta$-blockers). Second, the a priori risk factors were further selected using a bootstrap stepwise procedure to determine which factors were actually associated with the outcomes of interest. ${ }^{11}$ Using this approach, 1000 replicated bootstrap samples were selected from the original cohort. A bootstrap sample is a sample of the same size as the original data set chosen with replacement. Thus, a given subject in the original cohort may be selected multiple times, only once, or not at all, in a specific bootstrap sample. A stepwise procedure, using thresholds of $\mathrm{p}=0.05$ for variable selection and elimination, was applied to each replicated sample, and only the risk factors selected in at least $50 \%$ of the procedures were included in the conditional logistic regression models as confounders. ORs and 95\% CIs were calculated with ' $0 \leq \mathrm{PDC} \leq 0.5$ ' defined as the reference group to which all other categories were compared.

\section{Time-independent sampling: description of the bias}

A major challenge in this type of observational study is that exposure and outcomes are both measured in the same time window. Consequently, the time of onset of an outcome affects the classification of the drug regimen. When exposure and outcome are measured within the same time window, patients exposed to drug treatment who experience early outcomes are more likely to be classified as adherent to treatment than those with no or late outcomes during follow-up. In fact, the probability of complying with drug therapy after an MI decreases over time. ${ }^{12}$ Consequently, using a timeindependent sampling, treated patients who become 'early cases' will have a greater chance of being classified as adherent. This happens because the density of prescriptions is very high immediately after the acute event and then declines towards intermittent or cyclical patterns of drug intake, or to definitive treatment discontinuation (figure 1). The reduction in adherence over time can produce an over-representation of adherent cases and an underestimate of any beneficial adherence effect. This systematic error can be termed 'change in adherence bias' and could be considered a special case of the time-window bias when the following conditions occur: the exposure is a measure of medication adherence, the observation period begins with an acute and traumatic event, the study medications are chronic drug therapies and the patients are followed for long periods.

\section{RESULTS}

Of the initial 9720 resident patients discharged alive after a first MI in the enrolment period who were aged 35-100 years at discharge, 6880 patients were enrolled in the study cohort (figure 2). Of these, $67.5 \%$ were men. The mean age was 72.5 years for women and 63.7 years for men. The median follow-up was 994.5 days. A total of 4764 patients were treated $(\mathrm{PDC}>0.75)$ with antiplatelet agents, 696 patients with $\beta$-blockers, 4289 patients with ACEI/ARBs and 4433 patients with statins. For each of the study medications (figure 3), the probability of being classified as 'adherent to treatment' decreased as the length of the observation period increased. This probability varied from $85 \%$ to slightly more than $40 \%$ for patients treated with antiplatelet agents, from $45 \%$ to $10 \%$ for users of $\beta$-blockers, from $80 \%$ to $50 \%$ for patients treated with ACEI/ARBs and from $85 \%$ to $55 \%$ for statins users.

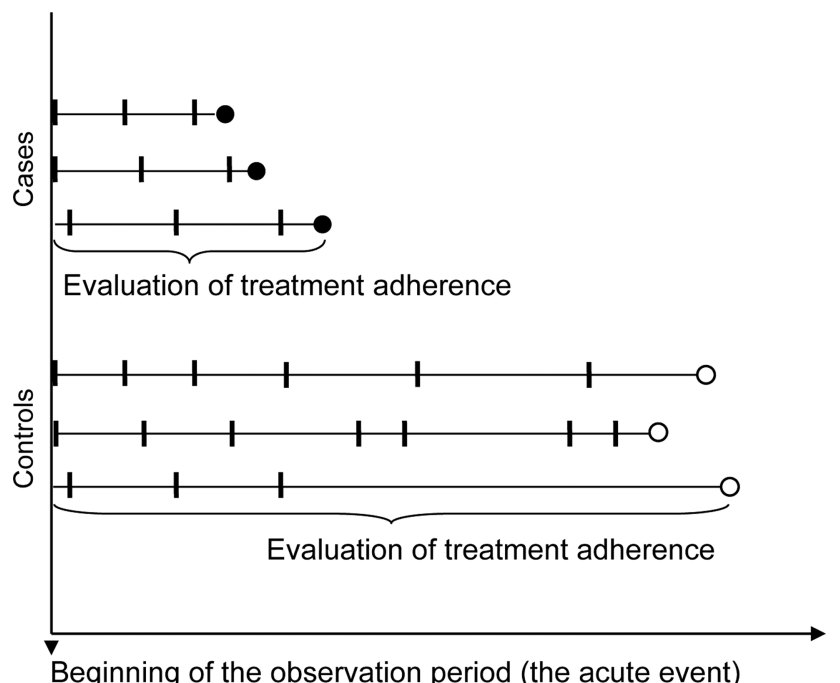

Vertical bars denote prescriptions filled.

Figure 1 Time-independent sampling: the change in adherence bias. 


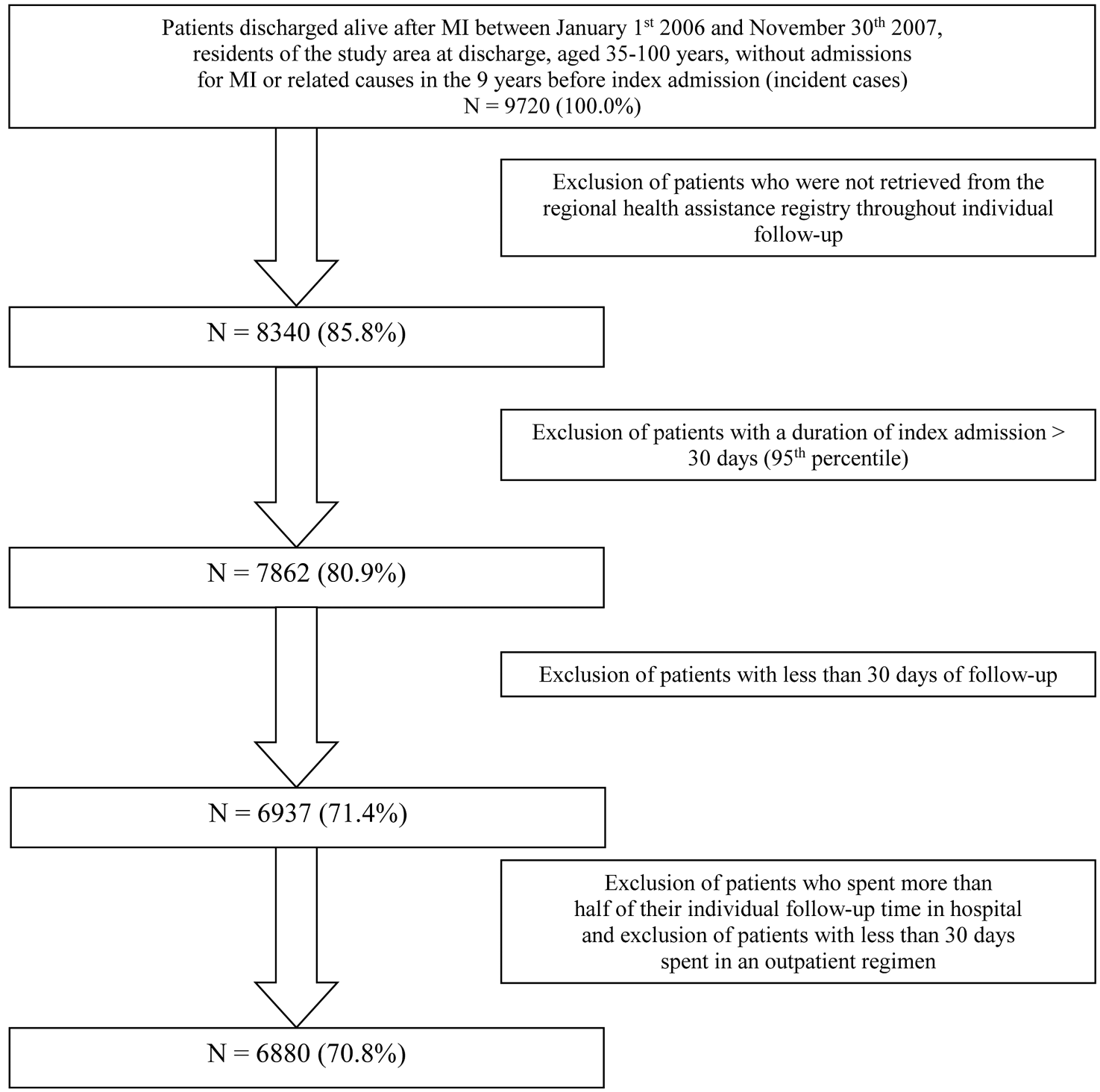

Figure 2 Cohort selection: flow chart. MI, myocardial infarction.

The nested case-control study was based on 778 cases of reinfarction. The time-independent cumulative incidence sampling produced a marked difference in the median observation times between cases and controls. Cases were observed for a median of 277 days and controls for a median of 1033 days.

The demographic and clinical characteristics of the nested case-control population derived from the timedependent sampling are reported in table 1.

In table 2 the strength of the association between the adherence to treatment and the incidence of a new MI is reported for each of the study drugs. Using the proper, unbiased, time-dependent sampling, a protective effect was detected for all study drugs. The risk of reinfarction decreased with increasing treatment coverage. The greatest benefits were observed with antiplatelet agents (relative risk reduction for 'PDC>0.75 vs $0 \leq \mathrm{PDC} \leq 0.5$ ': $37 \%$ ) and $\beta$-blockers (relative risk reduction for 'PDC $>0.50$ vs $0 \leq \mathrm{PDC} \leq 0.25$ ': $32 \%$ ). Based on the
ORs, a benefit was also evident for statins, but did not reach statistical significance.

The use of time-independent sampling produced very different results. The protective effect of the study drugs was largely attenuated (for antiplatelet agents and statins) or completely disappeared (for $\beta$-blockers and ACEI/ARBs). None of the associations were statistically significant. Furthermore, for all the study drugs, the risk of reinfarction increased with increasing adherence, from 'intermediate' to the 'higher' adherence category; this trend was the reverse of that observed using timedependent sampling.

\section{DISCUSSION}

In cohort studies where exposure and outcomes are measured in the same time-window, the time of the onset of an outcome affects drug regimen classification. Similarly, in case-control studies nested within a cohort, the choice 

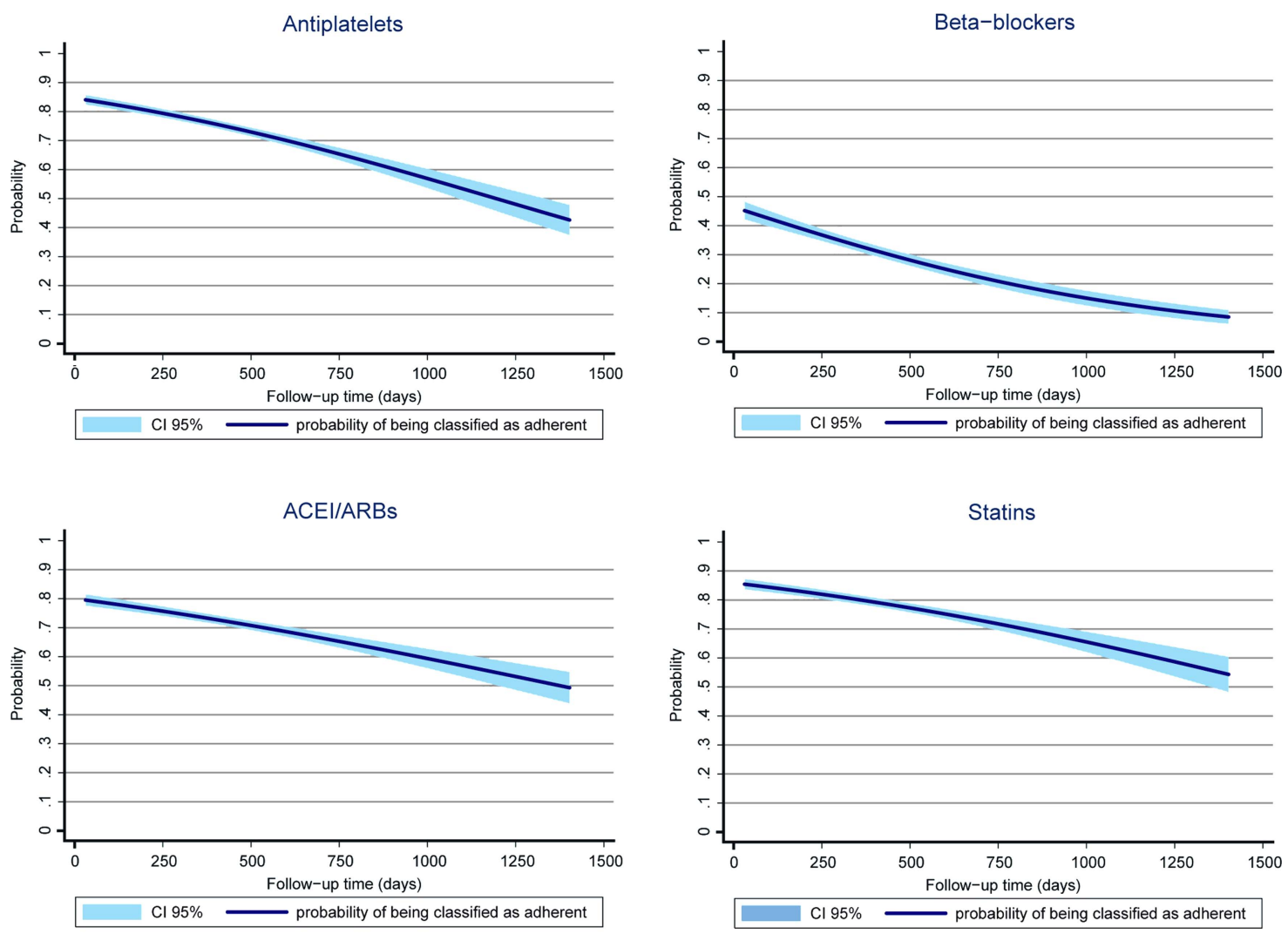

Analyses were performed separately for patients treated with each specific study drug

Figure 3 Probability of being classified as 'adherent to treatment' by follow-up time, starting from the date of discharge of the index myocardial infarction. ACEI, ACE-inhibitors; ARBs, angiotensin receptor blockers.

of the time-window in which exposure is measured is fundamental to the production of unbiased estimates.

Suissa has already demonstrated the importance of ensuring an identical time to measure exposure for cases and controls. If this condition is not met, the 'time-window bias', an error that is still frequent in observational research, will usually result in a biased estimate of the exposure effect. ${ }^{2}$

In clinical practice, medication non-adherence is one of the main factors that reduce the effectiveness of drug therapies; ${ }^{1}$ therefore, the aim of this study was to evaluate the impact of the time-window bias when the exposure is a measure of medication adherence. In this setting, we found that this bias produces an underestimate of the beneficial effect of good medication adherence that conforms to clinical guidelines.

In the cohort of patients with a first acute MI, as the length of the follow-up increased, the probability of a patient being classified as adherent to evidence-based treatments progressively decreased. This trend usually occurs when the observation period begins with an acute and traumatic event, and the study medications are chronic, secondary prevention therapies. ${ }^{12}$ Because exposure time overlaps follow-up time, patients who have already begun therapy and experience early outcomes during the exposure measurement period are more likely to be classified as adherent to treatment. In a nested study, if controls are selected using timeindependent sampling, this 'differential misclassification' produces an over-representation of adherent cases, which introduces a bias and an underestimation of the protective effect of medication adherence. We measured the impact of this 'change in adherence' bias by analysing the effect of evidence-based therapies on the incidence of a new MI. By using the proper time-dependent sampling, a protective effect was clearly detected for all study drugs, confirming the results of clinical trials and strengthening their validity in clinical practice. ${ }^{4513}$

Conversely, the use of a time-independent sampling spuriously attenuated the beneficial effect of antiplatelet agents and statins, and completely masked the beneficial effect of ACEI/ARBs and $\beta$-blockers. For this latter class of drug, we used lower PDC thresholds $(0.25$ and 0.5 vs 0.5 and 0.75 ) than used for the other drug groups. In fact, calculating drug coverage using the DDD carries the risk of not accounting for real-life dosing when a drug is used for indications different from its principal indication. This discrepancy is particularly relevant for $\beta$-blockers, which are usually prescribed at dosages lower than the DDD for secondary prevention after an MI. Unfortunately, information on daily doses prescribed to individual patients was not available. As a last remark, we 
Table 1 Demographic and clinical characteristics of the nested case-control population derived from the time-dependent sampling

\begin{tabular}{lll}
\hline & Cases & Controls \\
\hline $\mathrm{N}$ & 778 & 3083 \\
Demographics & 76 & 76 \\
$\quad$ Age (median-years) & 59.4 & 59.4 \\
$\quad$ Gender (\% male) & $284 ; 36.5$ & $1482 ; 48.0$ \\
Revascularisation procedures during the index admission & $6 ; 0.8$ & $75 ; 2.4$ \\
PCl (N; \%) & & \\
Bypass (N; \%) & $96 ; 12.3$ & $309 ; 10.0$ \\
Comorbidities (index admissions and 9 years before) & $248 ; 31.9$ & $488 ; 15.8$ \\
Malignant neoplasm (N; \%) & $141 ; 18.1$ & $362 ; 11.7$ \\
Diabetes (N; \%) & $113 ; 14.5$ & $265 ; 8.6$ \\
Disorders of lipid metabolism/obesity (N; \%) & $404 ; 51.9$ & $1119 ; 36.3$ \\
Hematological diseases (N; \%) & $92 ; 11.8$ & $338 ; 11.0$ \\
Hypertension (N; \%) & $263 ; 33.8$ & $749 ; 24.3$ \\
Conduction disorders (N; \%) & $279 ; 35.9$ & $590 ; 19.1$ \\
Cardiac dysrhythmias (N; \%) & $199 ; 25.6$ & $501 ; 16.3$ \\
Heart failure (N; \%) & $186 ; 23.9$ & $522 ; 16.9$ \\
Other cardiac diseases (N; \%) & $389 ; 50.0$ & $830 ; 26.9$ \\
Cerebrovascular disease (N; \%) & $146 ; 18.8$ & $334 ; 10.8$ \\
Diseases of arteries, arterioles and capillaries (N; \%) & $180 ; 23.1$ & $308 ; 10.0$ \\
Chronic obstructive pulmonary disease (N; \%) & $46 ; 5.9$ & $126 ; 4.1$ \\
Chronic nephropathies (N; \%) & $28 ; 3.6$ & $53 ; 1.7$ \\
Chronic liver, pancreas, digestive diseases (N; \%) & \\
Gastro-oesophageal haemorrhage (N; \%) & $269 ; 34.6$ & $802 ; 26.0$ \\
Evidence-based drug use during the 12 months before admission (at least 2 prescriptions) & $334 ; 10.8$ \\
Antiplatelet (N; \%) & $97 ; 12.5$ & $1412 ; 45.8$ \\
$\beta$-Blockers (N; \%) & $374 ; 48.1$ & $350 ; 11.4$ \\
ACE-inhibitors/ARBs (N; \%) & $126 ; 16.2$ &
\end{tabular}

ARBs, angiotensin receptor blockers; $\mathrm{PCl}$, percutaneous coronary intervention.

Table 2 Association between the levels of adherence to evidence-based drug therapies for secondary prevention after an acute myocardial infarction (MI) and the incidence of a new MI: a comparison between 'time-dependent' and 'time-independent' sampling methods

\begin{tabular}{|c|c|c|c|c|c|c|}
\hline & \multicolumn{3}{|c|}{ Time-dependent sampling } & \multicolumn{3}{|c|}{ Time-independent sampling } \\
\hline & OR & $\mathrm{Cl}$ 95\% & p Value & OR & $\mathrm{Cl} 95 \%$ & p Value \\
\hline \multicolumn{7}{|l|}{ Antiplatelet agents } \\
\hline $0 \leq \mathrm{PDC} \leq 0.5$ & 1.00 & - & - & 1.00 & - & - \\
\hline $0.5<\mathrm{PDC} \leq 0.75$ & 0.82 & 0.59 to 1.16 & 0.261 & 0.81 & 0.56 to 1.16 & 0.245 \\
\hline PDC>0.75 & 0.63 & 0.48 to 0.84 & 0.001 & 0.93 & 0.69 to 1.25 & 0.618 \\
\hline \multicolumn{7}{|l|}{$\beta$-Blockers } \\
\hline $0 \leq \mathrm{PDC} \leq 0.25$ & 1.00 & - & - & 1.00 & - & - \\
\hline $0.25<\mathrm{PDC} \leq 0.5$ & 0.95 & 0.70 to 1.28 & 0.722 & 1.11 & 0.82 to 1.51 & 0.508 \\
\hline$P D C>0.5$ & 0.68 & 0.49 to 0.94 & 0.018 & 1.23 & 0.89 to 1.72 & 0.211 \\
\hline \multicolumn{7}{|c|}{ ACE-inhibitors/ARBs } \\
\hline $0 \leq \mathrm{PDC} \leq 0.5$ & 1.00 & - & - & 1.00 & - & - \\
\hline $0.5<\mathrm{PDC} \leq 0.75$ & 0.83 & 0.57 to 1.21 & 0.343 & 0.96 & 0.65 to 1.43 & 0.858 \\
\hline PDC>0.75 & 0.72 & 0.55 to 0.95 & 0.018 & 1.00 & 0.76 to 1.33 & 0.977 \\
\hline \multicolumn{7}{|l|}{ Statins } \\
\hline $0 \leq \mathrm{PDC} \leq 0.5$ & 1.00 & - & - & 1.00 & - & - \\
\hline $0.5<\mathrm{PDC} \leq 0.75$ & 0.79 & 0.48 to 1.31 & 0.367 & 0.78 & 0.47 to 1.28 & 0.326 \\
\hline PDC>0.75 & 0.76 & 0.55 to 1.05 & 0.100 & 0.86 & 0.62 to 1.20 & 0.376 \\
\hline
\end{tabular}


used the incidence density sampling for nested casecontrol analyses specifically to measure the impact of time-window bias, which is specific to case-control studies. When the effect of medication adherence on health outcomes is estimated from a cohort study, models with time dependent covariates ${ }^{14}$ or marginal structural models ${ }^{15}$ are recommended.

\section{CONCLUSION}

When the exposure of interest is adherence to chronic medication, measured during a lengthy observation period that begins with an acute and traumatic event, the selection of controls using time-independent sampling may underestimate any beneficial adherence effect. The bias that we have described is not restricted to case-control studies. In the cohort as well as in the case-control study designs, the probability of a timedependent exposure changes with the length of follow-up. Therefore, time-independent exposure definitions will introduce a bias when the duration of follow-up varies with the outcome. The persistence of this type of time-related bias in peer-reviewed publications ${ }^{16-18}$ confirms the need for increased awareness of this methodological pitfall. ${ }^{19}$

Contributors MDM contributed to the concept and design of the study, the analysis of data, the interpretation of results and the writing of the article. UK, NA and DF contributed to the design of the study, the interpretation of results and the writing of the article. LB contributed to the design of the study, the acquisition of data from the health information systems, the statistical analysis, the interpretation of results and the writing of the article. MD and CAP contributed to the concept of the study, the interpretation of results, the writing of the article and the critical revision of the paper for important intellectual content, and they have given their final approval of the version submitted for publication. All authors agree to be accountable for all aspects of the work and ensure that questions related to the accuracy or integrity of any part of the work are appropriately investigated and resolved.

Funding This research received no grant from any funding agency in the public, commercial, or not-for-profit sectors.

Competing interests None declared.

Ethics approval This study was carried out in full compliance with the current privacy laws. The Department of Epidemiology is legitimised by the Lazio Region Committee in managing and analysing data from the regional health information systems for epidemiological purposes.

Provenance and peer review Not commissioned; externally peer reviewed.

Data sharing statement No additional data are available.

Open Access This is an Open Access article distributed in accordance with the Creative Commons Attribution Non Commercial (CC BY-NC 4.0) license, which permits others to distribute, remix, adapt, build upon this work noncommercially, and license their derivative works on different terms, provided the original work is properly cited and the use is non-commercial. See: http:// creativecommons.org/licenses/by-nc/4.0/

\section{REFERENCES}

1. Marcum ZA, Sevick MA, Handler SM. Medication nonadherence: a diagnosable and treatable medical condition. JAMA 2013;309:2105-6.

2. Suissa S, Dell'Aniello S, Vahey S, et al. Time-window bias in case-control studies. Statins and lung cancer. Epidemiology 2011;22:228-31.

3. Rothman KJ, Greenland S. Case-control studies. In: Rothman KJ, Greenland S, eds. Modern epidemiology. 2nd edn. Philadelphia: Lippincott Williams \& Wilkins, 1998:93-114.

4. Antman EM, Hand M, Armstrong PW, et al. 2007 focused update of the ACC/AHA 2004 Guidelines for the management of patients with ST-elevation myocardial infarction: a report of the American College of Cardiology/American Heart Association Task Force on Practice Guidelines (Writing Group to Review New Evidence and Update the ACC/AHA 2004 Guidelines for the Management of Patients With ST-Elevation Myocardial Infarction). Circulation 2008;117:296-329.

5. Cooper A, Skinner J, Nherera L, et al. Clinical guidelines and evidence review for post myocardial infarction: secondary prevention in primary and secondary care for patients following a myocardial infarction. NICE Clinical Guidelines, No. 48, London: National Collaborating Centre for Primary Care and Royal College of General Practitioners, 2007.

6. Kirchmayer U, Agabiti N, Belleudi V, et al. Socio-demographic differences in adherence to evidence-based drug therapy after hospital discharge from acute myocardial infarction: a population-based cohort study in Rome, Italy. J Clin Pharm Ther 2012;37:37-44.

7. Hammar N, Alfredsson L, Rosén M, et al. A national record linkage to study acute myocardial infarction incidence and case fatality in Sweden. Int J Epidemiol 2001;30:S30-4.

8. Belleudi V, Fusco D, Kirchmayer U, et al. Definition of patients treated with evidence based drugs in absence of prescribed daily doses: the example of acute myocardial infarction. Pharmacoepidemiol Drug Saf 2011;20:169-76.

9. Kirchmayer U, Di Martino M, Agabiti N, et al. Effect of evidence-based drug therapy on long-term outcomes in patients discharged after myocardial infarction: a nested case-control study in Italy. Pharmacoepidemiol Drug Saf 2013;22:649-57.

10. Suissa S. Immeasurable time bias in observational studies of drug effects on mortality. Am J Epidemiol 2008;168:329-35.

11. Austin PC, Tu JV. Automated variable selection methods for logistic regression produced unstable models for predicting acute myocardial infarction mortality. J Clin Epidemiol 2004;57:1138-46.

12. Filippi A, D'Ambrosio G, Giustini SE, et al. Pharmacological treatment after acute myocardial infarction from 2001 to 2006: a survey in Italian primary care. J Cardiovasc Med 2009;10:714-18.

13. Vanuzzo L, Pilotto L, Pilotto L, et al., For the WHO MONICA Project. Pharmacological treatment during $\mathrm{Ml}$ and in secondary prevention: the scientific evidence. World Health Organization (WHO) and the WHO MONICA Project investigators, 2000. http://www.ktl.fi/ publications/monica/carpfish/appenda/evidence.htm

14. Stricker BH, Stijnen T. Analysis of individual drug use as a timevarying determinant of exposure in prospective population-based cohort studies. Eur J Epidemiol 2010;25:245-51.

15. Hernán MA, McAdams M, McGrath $\mathrm{N}$, et al. Observation plans in longitudinal studies with time-varying treatments. Stat Methods Med Res 2009;18:27-52.

16. Khurana V, Bejjanki HR, Caldito $G$, et al. Statins reduce the risk of lung cancer in humans: a large case-control study of US veterans. Chest 2007;131:1282-8.

17. Suissa S. Immortal time bias in pharmaco-epidemiology. Am J Epidemiol 2008;167:492-9.

18. Suissa S. Randomized trials built on sand: examples from COPD, hormone therapy, and cancer. Rambam Maimonides Med J 2012;3: e0014.

19. Hernández-Díaz S. Name of the bias and sex of the angels. Epidemiology 2011;22:232-3. 